\section{SCIENCE CHINA \\ Physics, Mechanics \& Astronomy}

- Editorial •

Editor's Focus
February 2020 Vol. 63 No. 2: 221061

https://doi.org/10.1007/s11433-019-1472-6

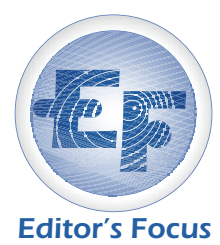

\title{
Exploring the doubly charmed baryon
}

The doubly charmed baryon, like the proton and neutron, is formed by three quarks. However, they are different, as such a baryon consists of two heavy quarks (charm-quark) and one light quark, while the proton and neutron respectively contain three light quarks. Therefore the doubly charmed baryon is expected to have different internal structures and properties. For example, its mass is 3-4 times higher than those of the proton and neutron. Studying the doubly charmed baryons can provide important information to understand the three-body bound system, the hadronic interaction and the quark model.

Recently, the LHCb collaboration [1] reported the observation of the doubly charmed baryon $\Xi_{c c}^{++}$(quark content: $c c u$ ) via its decay to $\Lambda_{c}^{+} K^{-} \pi^{+} \pi^{+}$final states and measured its mass. The LHCb collaboration also measured the $\Xi_{c c}^{++}$lifetime [2], and established the weak decay nature of the doubly charmed baryon. The observation of the $\Xi_{c c}^{++}$baryon has been confirmed in another decay mode $\Xi_{c c}^{++} \rightarrow \Xi_{c}^{+} \pi^{+}$by the LHCb collaboration [3].

In the current work [4], the LHCb collaboration reported the results of a search for the doubly charmed baryon $\Xi_{c c}^{+}$(quark content $c c d$ ), which is the isospin partner of the $\Xi_{c c}^{++}$baryon. They have observed some piece of evidence (hint) of the signal at the same place of the $\Xi_{c c}^{++}$mass with a local (global) significance of $3.1 \sigma(1.7 \sigma)$. They reported the most stringent upper limits on the ratio of the production rate of the $\Xi_{c c}^{+}$baryon relative to that of the $\Xi_{c c}^{++}$baryon and the singly charmed baryon $\Lambda_{c}^{+}$. This is an important milestone to the observation of the singly charged $\Xi_{c c}^{+}$.

1 R. Aaij, et al. (LHCb Collaboration), Phys. Rev. Lett. 119, 112001 (2017).

2 R. Aaij, et al. (LHCb Collaboration), Phys. Rev. Lett. 121, 052002 (2018).
3 R. Aaij, et al. (LHCb Collaboration), Phys. Rev. Lett. 121, 162002 (2018).

4 R. Aaij, et al. (LHCb Collaboration), Sci. China-Phys. Mech. Astron. 63, 221062 (2020). 